\title{
Explore: The Value of Prostatic Calculi in Prostate Surgery
}

\author{
Xiao Chuan, Li Luhong, Wang Yong, Yi Zhengjin, Liu Xiangyu, Li Suqiong, Cao Jingzhao, \\ Tang Ruipeng, Li Xin, Wan Li
}

Department of Urology, Pangang Group General Hospital, Panzhihua, China

Email address:

260649926@qq.com (Xiao Chuan),413862432@qq.com (Li Luhong),1055657038@qq.com (Wang Yong), 507514836@qq.com (Yi Zhengjin),3058595604@qq.com (Liu Xiangyu),41923483172@qq.com (Li Suqiong),

704145030@qq.com (Cao Jingzhao),459477817@qq.com (Tang Ruipeng),171659633@qq.com (Li Xin),446247758@qq.com (Wan Li)

${ }^{*}$ Corresponding author

\section{To cite this article:}

Xiao Chuan, Li Luhong, Wang Yong, Yi Zhengjin, Liu Xiangyu, Li Suqiong, Cao Jingzhao, Tang Ruipeng, Li Xin, Wan Li. Explore: The Value of Prostatic Calculi in Prostate Surgery. International Journal of Clinical Urology. Vol. 4, No. 1, 2020, pp. 21-24.

doi: $10.11648 /$ j.ijcu.20200401.15

Received: October 9, 2019; Accepted: December 11, 2019; Published: March 24, 2020

\begin{abstract}
Objective To explore the significance of prostatic calculi in prostate surgery. Methods During the beginning of 2010 to the end of 2017, a retrospective analysis was made of 821 cases of benign prostatic hyperplasia (BPH) with prostatic calculi admitted to our hospital. During 2010 to 2015, transurethral resection of the prostate (TURP) was performed. And then, from 2015 to 2017, it was replaced as transurethral laser vaporization of the prostate (TULVP). There were 5 converted to open. We should remove the prostate tissue til the surgical capsule by electrocision or vaporization. If annular fibrous tissue or prostatic calculi are encountered during the operation, the calculi will be exfoliated, and then stop operation. Results All the operations were successfully completed, with an average times of 47 minutes (22-117 minutes), bleeding of 50ml (15-350 ml), indwelling catheter for 5.7 days (3-10 days), and continuous bladder irrigation for 1.3 days (1-5 days). During the follow-up period of 0.4-5 years, the urination symptoms and quality of life of the post-operation patients were significantly improved. Conclusions Prostate calculi can be used as a marker of the depth of operation for BPH.
\end{abstract}

Keywords: Prostate Calculi, $1470 \mathrm{~nm}$ Laser, TURP, TULVP, Operative Sign

\section{Introduction}

From the beginning of 2010 to the end of 2017, there were 1558 benign prostatic hyperplasia (BPH) patients who treated in our hospital. Among them, 821 patients were treated surgically with prostatic calculi. During 2010 to 2015, transurethral resection of the prostate (TURP) was performed. And then, from 2015 to 2017, it was replaced as transurethral laser vaporization of the prostate (TULVP). The summary report is as follows.

\section{Clinical Materials and Methods}

\subsection{General Information}

The average age of 821 patients was 69.7 years (51-93 years). The duration of dysuria was 1-10 years. The average International Prostate Symptom Score (IPSS) were 23.7 points
(17-25 points). Rectal digital examination and prostate ultrasonography indicated that $\mathrm{BPH}^{\circ}{ }^{\circ}-\mathrm{III}^{\circ}$, prostate weight 40-150 g, residual urine volume $30-130 \mathrm{ml}$ ". Or he had the history of urinary retention, or the maximum urinary flow rate (Qmax) was 3.2-13ml/s. The mean prostate specific antigen (PSA) was $3.5 \mathrm{ng} / \mathrm{ml}(0.4-9.6 \mathrm{ng} / \mathrm{ml})$. All patients who's PSA ranging from 4 to $10 \mathrm{ng} / \mathrm{ml}$ underwent prostate biopsy, and biopsy confirmed non-prostate cancer. Among 821 cases, 453 cases were found prostate stones by abdominal ultrasound or pelvic CT before operation, while 368 cases were found during operation.

Indications for prostate surgery: 1) Elderly men (age $>55$ years old); 2) Those with severe symptoms, age can be relaxed to 50 years old; 3) Prostate cancer has been excluded; 4) Dysuria symptoms are obvious, affecting life for more than one year, and conservative treatment is not good; 5) IPSS $>17$ points, prostate size II-III ${ }^{\circ}$; 6) Combined with bladder diverticulum or bladder stones; 7) Renal insufficiency caused 
by lower urinary tract obstruction; 8) Chronic diseases such as heart, lung, brain or blood have been controlled.

\subsection{Method}

TULVP or TURP were performed by electrotomy mirror (Wolf) and $1470 \mathrm{~nm}$ laser (Wuhan Qizhi). General anesthesia was used. After sterilization and towel laying, $26 \mathrm{Fr}$ cystoscope sheath was inserted through the urethra (urethral stricture patients need urethral dilatation first). Cystoscopy was inserted through sheath to observe whether there was neoplasm and cystic calculus, and then found the bilateral ureteral openings. The cystoscope was retracted to the bladder neck and evaluated the distance between the bladder neck to the spermatorrhea. The prostate tissue was removed from the middle lobe at 5 and 7 o'clock with a cutting ring or $1470 \mathrm{~nm}$ laser, then the bilateral lobes and the top of the prostate were removed. Finally, the tip of the prostate was removed. If thick and sparse annular fibrous bundles (prostatic capsule) or prostatic calculi are found during the operation, the operation will be discontinued. If prostatic calculi are found during the operation, they will be removed completely and pushed into the bladder. The bleeding and hemostasis were observed in the operation area, and the tissue mass and fragments were sucked out. After ensured no active bleeding, once again, $20 \mathrm{Fr}$ three-chamber balloon urethral catheter was inserted, and the balloon was injected with $50 \mathrm{ml}$ of water. The bladder was continuously washed with normal saline.

\subsection{Statistics of Perioperative Conditions and Complications}

Among the 821 patients, 527 were treated with TURP from 2010 2015, 289 were treated with TULVP from 2015 2018, and 5 were converted to open (Conversion to open was also considered as complete case.). All patients completed the operation smoothly, the operation time was about 22-117 minutes (average of 47 minutes); Bleeding was about 15-350 $\mathrm{ml}$ (average of $50 \mathrm{ml}$ ); Indwelling catheter for 3-10 days (average of 5.7 days); Continuous bladder irrigation for 1-5 days (average of 1.3 days).

Follow-up for 0.4-5 years showed that complications were as follows: 1) 253 patients with urinary tract irritation symptoms and urinary tract infection were cured after the use of effective antibiotics and $\alpha$-blockers; 2) 22 patients with delayed bleeding after operation, in 21 of them whom were cured after bladder clot clearance by cystoscopy, and another one patient was cured after intravesical blood clot removal and prostate hemostasis under general anesthesia; 3) 78 cases of stricture of the external orifice of urethra and bladder neck were cured within 3 months after operation. All cases were cured after regular urethral dilatation. 4) 15 cases of transurethral resection syndrome (TURS) appeared in perioperative period. And after timely treatment, such as diuresis (furosemide) and electrolyte supplementation (sodium ion), they were controlled; 5) because of the huge prostatic gland, there were 3 consecutive patients undergoing secondary prostate surgery; 6) 17 cases of pseudo incontinence occurred after operation, which were cured within six months through anal lifting training and penile clip training; 7) 1 case of genuine incontinence, and 2 cases of genuine incontinence. Anterior urinary incontinence was improved (penile clips were used during the day and urination was self-evacuating after urine storage at night).

No other systemic diseases were aggravated and no perforation, rectal injury or death occurred. Postoperative IPSS decreased by 5.6 points on average. Residual urine volume was $0-20 \mathrm{ml}$, Qmax improved by $17.6 \mathrm{ml} / \mathrm{s}$ on average. The micturition symptoms and quality of life of the above patients were significantly improved after operation.

\section{Results}

Through the analysis and comparison of our hospital's 821 cases and Xiao Congqin [1] (Guangzhou First People's Hospital Urology) statistics of 390 cases of BPH patients by TURP treatment. SPSS 13.0 software was used for statistical analysis. The difference was statistically significant with $\mathrm{P}<0.05$. The data of perioperative and postoperative complications of patients with benign prostatic hyperplasia and prostatic calculi in our hospital were significantly different from those of Guangzhou First People's Hospital $(\mathrm{P}<0.05)$.

Table 1. The data of two groups.

\begin{tabular}{|c|c|c|c|c|c|}
\hline & Operative time & Intraoperative hemorrhage & perioperative complications & Postoperative IPSS & Qmax \\
\hline $\begin{array}{l}\text { Guangzhou First People's } \\
\text { Hospital }(n=390)\end{array}$ & $\begin{array}{l}30 \sim 130 \mathrm{~min} \\
(52 \pm 1.9 \mathrm{~min})\end{array}$ & $50 \sim 200 \mathrm{ml}(100 \pm 10.4 \mathrm{ml})$ & 50 & $6.3 \pm 1.9$ & $20.9 \pm 2.3 \mathrm{ml} / \mathrm{s}$ \\
\hline $\begin{array}{l}\text { Pangang Group General } \\
\text { Hospital }(n=821)\end{array}$ & $\begin{array}{l}22 \sim 117 \mathrm{~min} \\
(47 \pm \min )\end{array}$ & $15 \sim 350 \mathrm{ml}(50 \pm \mathrm{ml})$ & 131 & 5.6 & $17.6 \mathrm{ml} / \mathrm{s}$ \\
\hline
\end{tabular}

Table 1. Continued.

\begin{tabular}{|c|c|c|c|c|}
\hline & Secondary hemorrhage & temporary incontinence & Genuine urinary incontinence & urethral stricture \\
\hline $\begin{array}{l}\text { Guangzhou First People's } \\
\text { Hospital }(n=390)\end{array}$ & 8 & 10 & 0 & 7 \\
\hline $\begin{array}{l}\text { Pangang Group General } \\
\text { Hospital }(n=821)\end{array}$ & 22 & 17 & 1 & 78 \\
\hline
\end{tabular}

With prostate stones as the boundary, prostatectomy was performed. The results were compared with the traditional TURP treatment in Guangzhou First People's Hospital. One case of genuine urinary incontinence after operation in our hospital was unable to form an effective comparison; the incidence of urethral stricture after operation in our hospital was 
higher $(\mathrm{P}<0.05)$; the other indicators were not significantly different $(\mathrm{P}>0.05)$; the surgical techniques in our hospital were basically the same as those in the advanced hospitals in China. However, prostatectomy with prostate calculi as its boundary hasn't had any adverse manifestations such as prostate perforation, rectal injury and bladder neck amputation.

\section{Discussion}

$\mathrm{BPH}$ is one of the most common urinary tract obstructive diseases in elderly men, and the incidence is positively correlated with age. TURP, as a mature and widely developed surgical method, is the standard method for BPH surgical management, also known as the "gold standard" [1]. Traditionally, the surgical boundary of TURP is bladder neck, spermatorrhea and surgical capsule. In order to avoid serious complications such as perforation and urinary incontinence, the surgeon should always be vigilant about the boundary. In recent years, the incidence of complications such as perforation and urinary incontinence after TURP is still high because of the unsharp and noneffective indication of the depth. Recent years, BPH patients with prostatic calculi treated in our hospital can effectively avoid the surgical cut-off because of using the prostate calculi as the surgical boundary. Therefore, there were no case of prostatic perforation or rectal injury occurred. All patients were satisfied with the clinical effect, urination symptoms and quality of life were effectively improved $[2,3]$.

\subsection{Etiology and Pathogenesis of Prostate Calculi}

The incidence of prostate calculi is increasing by years, but its pathogenesis is still unclear. Zhao Guoxian et al. [4] analyzed 4323 physical examinees of different age groups. The incidence of prostate calculi was $32.03 \%$. The incidence of prostate stones was $48.91 \%$ in the group over 50 years old, and $68.52 \%$ in the group with BPH. Shao Qiang et al. [9] reported that transrectal ultrasonography of the prostate revealed that the incidence of prostate stones was $75 \%$ in middle-aged people and $100 \%$ in the elderly. This is one of the important dangerous signals affecting the quality of life and physical and mental health of elderly men [11].

There are many theories about the mechanism of prostate calculi. The most representative viewpoint is that the prostate calculi are caused by the concentration of prostatic fluid [5]. Others also believe that the prostate calculi are caused by the chronic inflammation of prostate acinar and excretory duct and salt deposits on prostate after urine reflux [6]. These views have not yet been confirmed. Recent studies have found that the unique mineralization of nanobacteria is the only pathogen that can produce apatite minerals and deposit in human body. Nanobacteria can cause a variety of organ lesions, and the infection rate is high in the population. The main way of excretion is the urinary system [7]. The position of prostate is close to the outlet of the urinary system. There are more chances of infection of nanobacteria, which may be related to the occurrence of prostate stones [12].

\subsection{Location of Prostate Stone Formation}

According to the relevant data, the location of prostatic calculi depends on the pathophysiology of prostate in different age groups [8]. (1) Young and middle-aged people ( $<50$ years old) are at high risk of prostatitis, and the prostatitis is closely related to the occurrence of prostatic calculi. This may be the reason why prostatitis can promote the deposition of calcium and phosphate in the prostate, stagnate secretions, obstruct glandular ducts and form stones. Therefore, the prostate stones in young and middle-aged patients are mostly distributed in the infection-prone areas of the internal glands, and are scattered in isolated single or dot-like forms. 2) The middle-aged and old people ( $>50$ years old) are the prone population of BPH. When $\mathrm{BPH}$ occurs, the internal glands are prominent, and the hyperplastic nodules of the internal glands squeeze the acinar ducts at the junction of the internal and external glands, making them dilate. Secretion stasis, glandular duct obstruction, to form stones. And middle-aged and old men with urinary weakness, residual urine and no easy to empty, and urinary reflux can also form stones. Therefore, most of the stones are located in the surgical capsule or the tip of the prostate at the junction of the internal and external glands, so they are densely clustered, either isolated or scattered [13].

\subsection{Exploration of Depth Markers for Transurethral Prostate Surgery}

In prostate surgery, we follow the principle of excision of glands between bladder neck and spermatorrhea, resection of thicker or sparser annular fibrous bundles at the junction of surgical capsule and gland, and the tip of prostate marked by spermatorrhea, which should not exceed the external urethral sphincter. However, perforation of the prostate and urinary incontinence is common in the actual operation [14]. Liu Wei [10] et al. summarized the clinical data of 616 cases of $\mathrm{BPH}$, and concluded that the "slippage phenomenon" is the appropriate depth. Because there is less hemoglobin in the annular fibrous bundle at the junction of surgical capsule as well as less glandular tissue on the surface. So that it has a higher resistance during electrotomy. This phenomenon called " slippage phenomenon ". The middle-aged and old-aged prostate stones are mostly located at the surgical capsule and the tip of the prostate at the junction of the internal and external glands. Therefore, electrotomy arriving at the prostate stones during the operation will also appear "slippery phenomenon".

However, with the improvement of surgical instruments, plasma electrotomy and $1470 \mathrm{~nm}$ laser prostatectomy can still work normally for hemoglobin-free bundles of fibers, so it is more likely to damage the bladder neck, urethral sphincter and rectum, so now "slippage phenomenon" can no longer be competent for the identification of prostate boundary [15]. Because the incidence of prostate calculi in middle-aged people is $75 \%$, and that in elderly people is $100 \%$, and prostate calculi are mostly located in the surgical envelope and the tip of the prostate, prostate calculi is used as one of the indicators to judge the depth of prostate resection in our hospital. When prostate calculi are found during operation, deep operation is 
stopped. The calculi are removed and slightly trimmed, then bilateral lobes are removed along the plane. At present, most medical institutions in China use enucleation of the prostate in urology, which separation is along the surgical envelope and the prostate gland. Most of the prostate stones in elderly patients are located in this area, so when enucleation, the prostate stones can still be used as a sign [16].

These patients were follow-up after discharge in our department. In general, the first telephone follow-up is completed within two weeks after discharge, and the second follow-up is completed within half a year. For patients with postoperative complications, the follow-up period can be extended to 5 years. Of the 821 patients, 794 completed at least one follow-up visit, while 27 lost follow-up due to phone number errors (these 27 patients were classified as the treatment group without complications). All patients were bounded by prostatic calculi, which effectively avoided cutting across the boundaries, and no prostatic perforation or rectal injury occurred. One patient had genuine urinary incontinence after operation. Consideration was given to the early enucleation of the prostate in our hospital, which was caused by immature surgical technique. At present, the urinary incontinence of the patient was improved significantly. The other patients were satisfied with the clinical effect, and the urination symptoms and quality of life were effectively improved after operation.

\subsection{Individual Case Sharing}

Five of the patients were converted to open: four of them were because of more bleeding in the wound. One case underwent urethral repair 20 years ago. It was found that the prostate was covered with stones, and the posterior urethra was rigid, and the bladder neck was elevated, and the sheath couldn't pass through, so the operation was transferred to open. The size of prostate was about $4 \times 3.5 \times 3 \mathrm{~cm}$ and the weight of prostate was about $100 \mathrm{~g}$. The symptoms of prostatic hypertrophy and the quality of life were improved significantly after operation.

\section{Conclusions}

In a word, after seven years of clinical observation, we analyzed the curative effect of TURP and TULVP in $821 \mathrm{BPH}$ patients with prostate calculi, and concluded that: TURP and TULVP can use prostate calculi as one of the indicators of the depth.

\section{References}

[1] Xiao CQ, Hu JB, Deng JH, et al. Transurethral resection of prostate in 390 high-risk patients with benign prostatic hyperplasia [J]. Acad J Guangzhou Med U, 2015, 43 (5): 56-58. DOI: $10.3969 /$ j.issn.2095-9664.2015.05.014.
[2] Tang ZG, Liang ZZ, Zhang YB, et al. Analysis of quality of life of patients with benign prostatic hyperplasia and its related influencing factors [J]. Chin J Clin (Electron), 2015, 13 (11): 2623-2626.

[3] Liu YC, Chen ZX, Pan X, et al. TURP for Treatment of High Risk Benign Prostatic Hyperplasia: A Report of 112 Cases [J]. Guid Chin Med, 2014, 12 (12): 31. 32.

[4] Zhao GX, Fu B, Wang HM, et al. The incidence of prostate stones in a city 4323 patients [J]. Guid Chin Med, 2012. 10 (18): 567-569.

[5] Fu Q, Wang FC, Guan LY. Clinical prostatology [M]. Ji Nan: Shangdong Science and Technology Press, 2008: 236-237.

[6] Qi HY, Zhu H. New progress in basic research and diagnosis and treatment of prostate diseases [M]. Beijing: People's Medical Publishing House, 2009: 193-196.

[7] Shen XC, Yang J, Rao XC, et al. Culture and morphological identification of nanobacteria from prostatic calculus [J]. Acta Acad Med Mil Terti, 2008, 30 (12): 1122-1124.

[8] Zhen F, Liu YH. Ultrasonographic characteristics of prostate stones in different age groups were observed and analyzed [J]. J Sourtheast Chin Nat Defence Med Sci, 2005, 7 (3): 204-205.

[9] Zhang YH, Shao Q. Prostate surgery [M]. Beijing: People's Medical Publishing House, 2001. 474-475.

[10] Liu W, Zhong HL, Chen J, et al. A discussion on signs of a proper depth of ablation during transurethral electrovaporization of the prostate [J]. Chin J Minim Invas Surg, 2005, 5 (7): 572-573.

[11] Sharma Ashish, Garg Gaurav, Sinha Rahul Janak, Singh Vishwajeet. Posterior urethral valve presenting with impacted prostatic urethral calculus: a diagnostic challenge $[\mathrm{J}]$. BMJ case reports, 2018, 2018.

[12] Shen XC, Yang J, Rao XC, et al. Nanobacteria: isolation from prostatic calculus and identification of its 16S rRNA gene [J]. Acta Acad Med Mil Terti, 2008 (02): 124-126.

[13] Lee Young Ju, Oh Seung-June. Calculi in the Prostatic Surgical Bed as a complication after Holmium Laser Enucleation of the Prostate [J]. Urol J, 2018. DOI: 10.22037/uj.v0i0.3965.

[14] Liu SW, Wang YD, Lin F. Preservation of $120^{\prime}$ clock position urethral mucosa in transurethral enucleation of the prostate reduced incontinence rate in patients with benign prostate hyperplasia [J]. J Minimal Invas Urol, 2018, 7 (01), 41-44. DOI: 10.19558/j.cnki.10-1020/r.2018.01.010.

[15] Sun JH. Comparative analysis of the efficacy and safety of transurethral laser vaporization of prostate 1470 and transurethral plasma resection of prostate in the treatment of BPH [J]. Cap food med, 2019, 26 (14): 46.

[16] Erika LK, Santiago GV, Gloria D, et al. Robot-assisted Radical Prostatectomy Due to a Primary Carcinoid Prostatic Tumor in a Three-Year-Old Child: A Case Report [J]. Urology, 2019, 133. DOI: 10.1016/j.urology.2019.08.006. 